\title{
Les prémices \\ du génome \\ de Néandertal
}

Simone Gilgenkrantz

\section{Neanderthal, la vallée de l'Autre}

En 1856, quand des ouvriers d'une carrière découvrirent pour la première fois en Allemagne, près de Düsseldorf, les restes fossilisés d'un «Homme de Neanderthal», la communauté scientifique n'imaginait guère les rebondissements à distance de cette découverte et de celle des autres sites qui suivirent, en Russie, Croatie, Belgique, Italie, à Rochers de Villeneuve [1], et à la Chapelle-aux-Saints en France.

Ces révélations venaient à point pour consolider le concept d'évolution du monde vivant qui commençait à s'imposer, malgré de sérieuses résistances des tenants du créationnisme. Puis au cours du XXe siècle, à l'étude morphologique comparative des paléontologues se substitua peu à peu la démarche analytique des biologistes moléculaires: I'analyse de l'ADN de deux espèces vivant aujourd'hui pouvait aider à établir leurs liens de parenté et à les situer sur l'arbre phylogénétique. Elle pouvait mesurer les changements survenus au cours de l'évolution, et proposer une reconstitution des chromosomes et de l'ADN de leur ancêtre commun.

Mais, à l'aube de la génétique moléculaire, l'analyse de I'ADN ancien provenant d'ossements ou de dents semblait inaccessible du fait des ravages du temps : la fragmentation, les réactions d'hydrolyse et de dégradation que peuvent subir ces molécules rendaient apparemment leur séquençage impossible.

Pourtant, dès la mise au point de la technique PCR (polymerase chain reaction), l'éventualité d'amplifier des séquences fragmentées d'ADN ancien fut envisagée. Les études de paléogénétique devinrent possibles et commencèrent à se multiplier. Elles ont permis de définir les positions phylogénétiques d'espèces disparues, comme par exemple le couagga [2, 3], ce bel équidé, entre le cheval et le zèbre, qui faisait la fierté

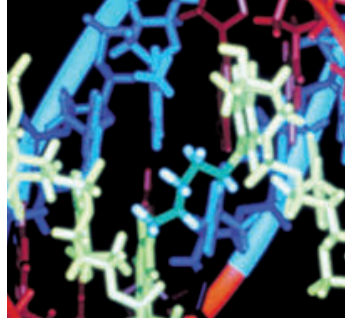

Médecine/Sciences,

9 , rue Basse,

54330 Clérey-sur-Brénon,

France.

sgilgenkrantz@

medecinesciences.org

des Écuries Royales de Versailles sous Louis XIV. Cette étude avait été faite à partir d'un animal taxidermisé et dans ce cas, comme dans celui de la momie égyptienne étudiée peu après [4], les conditions de conservation avaient été favorables et les spécimens ne dataient que de quelques centaines ou quelques milliers d'années. Ensuite, la remontée dans le temps devint vertigineuse : une séquence d'ADN de chloroplaste fut isolée à partir de feuilles de magnoliacée fossile datant du miocène (17 millions d'années), sédimentées en anaérobiose dans le gisement de Clarkia en Idaho (États-Unis). II sembla même ne plus y avoir de limites à cette ascension dans le temps, puisque furent publiées d'autres analyses de séquences remontant à des dizaines de

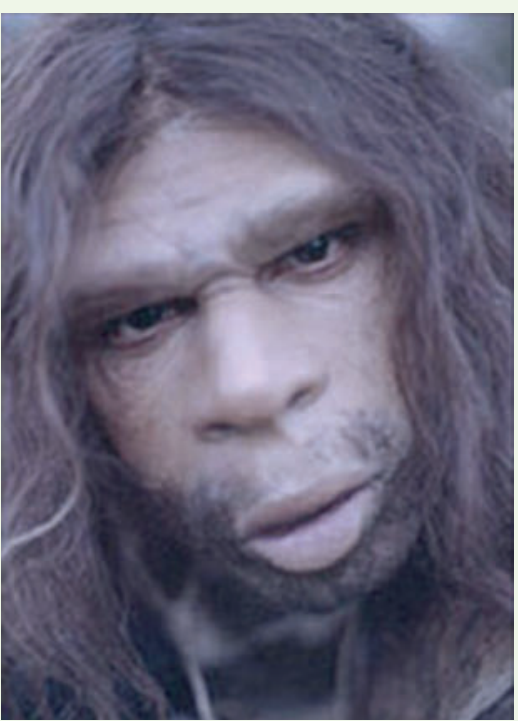


millions d'années: celles d'un charançon conservé dans l'ambre et même d'un dinosaure juste au moment où le premier Jurassic Park sortait sur les écrans!

\section{Faut pas rêver}

L'annonce fut accueillie avec scepticisme. Le paléogénéticien suédois Svante Pääbo jugeait nécessaire une confirmation par d'autres laboratoires. Très vite, il s'avéra que la séquence du charançon n'était autre que celle d'un banal champignon moderne, et celle de l'animal préhistorique un (presque inévitable) contaminant humain. Ce qui fut appelé «la débâcle des dinosaures » eut au moins le mérite d'attirer l'attention sur un problème majeur avec la PCR, surtout en paléontologie: le danger de contamination. Apparut alors la nécessité de prendre les plus extrêmes précautions, surtout s'agissant de séquences proches de l'homme moderne, puisqu'on ne pouvait s'affranchir de la principale source de contamination, par la présence des archéologues, biologistes ou tout autre humain s'approchant du fossile, depuis son exhumation sur le site des fouilles jusqu'au laboratoire. Des règles très strictes furent établies pour limiter toute contamination dans des codes de bonne pratique. C'est pourquoi le spectacle que donnent désormais les paléontologues en action évoque un peu les cosmonautes ou les chirurgiens en train d'opérer (blouses, masques, gants, matériel stérile...), les «pièces opératoires » étant immédiatement congelées.

Pourtant, avant toutes ces recommandations, S. Pääbo réussit en 1997 à décoder 379 bases d'ADN mitochondrial (ADNmt) à partir de 11 spécimens d'Homo neanderthalensis $[5,6]$. Travaillant avec son équipe à Münich (Allemagne), il montrait que ce dernier était une espèce distincte d'Homo sapiens et que la séparation entre les deux espèces pouvait remonter à un demi million d'années. Ces conclusions ont été confirmées et enrichies ensuite par d'autres chercheurs, en particulier tout récemment par l'équipe de Catherine Hänni, du laboratoire Paléogénétique et Évolution moléculaire de Lyon, à partir d'une dent d'un juvénile néandertalien, datant de 100000 ans [7].

\section{Une longue marche}

Séquencer de I'ADN nucléaire d'Homo neanderthalensis était encore plus délicat. La taphonomie ${ }^{1}$ nous apprend beaucoup sur la dégradation des tissus au cours de la diagenèse. À travers les siècles et les variations climatiques, l'état de préservation de l'ADN dépend de nombreux facteurs : température, humidité, $\mathrm{pH}$, agents oxydants... Et aucun spécimen de Néandertal n'a eu la chance d'être conservé dans un permafrost, - comme Ötzi, ce chasseur des glaces autrichien momifié, d'il y a 5000 ans, retrouvé en 2003 [8], le permafrost ou permagel (sol gelé en profondeur et en permanence) étant idéal pour une bonne conservation.

${ }^{1}$ de taphos : tombeau, science qui étudie les processus de dégradation des fossiles.
De surcroît, les spécimens ne sont pas nombreux, et les conservateurs de muséums sont peu enclins à autoriser des prélèvements sur du matériel aussi exceptionnel.

Aussi, S. Pääbo procéda par étapes: d'abord s'assurer sur 150 mg de l'intégrité des protéines, puis qu'il s'agissait bien d'ADN d'Homo neanderthalensis. Les résultats étant concluants, un prélèvement plus important fut réalisé sur un os appartenant à un spécimen de Néandertal datant de 38000 ans, et provenant de Vindjia, en Croatie.

Afin de pouvoir tirer un maximum de conclusions, deux techniques furent utilisées en parallèle dans deux laboratoires:

- L'équipe de S. Pääbo au Max Planck Institute for evolutionary anthropology, à Leipzig (Allemagne) a utilisé, grâce à l'emploi de la plate-forme 454 de la société de biotechnologie Life Sciences une technique de séquençage à très haut débit évitant le clonage des segments d'ADN à analyser [9]. Le séquenceur GS 20 (Genome Sequencer 20 mégabases) est capable de séquencer jusqu'à 20 mégabases par run de 45 heures.

- L'équipe de E.M. Rubin [10] utilise les techniques de clonage et de séquençage classiques, suivies d'une méthode sophistiquée d'analyse des séquences (par voie expérimentale et bio-informatique) mise au point sur des communautés microbiennes [11, 12] au Joint Genome Institute à Walnut Creek (CA, États-Unis), et adapté à l'ADN ancien par une analyse réussie d'ADN d'un ours des cavernes [13].

\section{Un début de décryptage}

\section{Qu'en est-il des premiers résultats?}

- S. Pääbo et al. réussissent à lire 1 million de paires de bases du génome d'Homo neanderthalensis. Comparé au génome du chimpanzé et de l'homme, quelques conclusions peuvent déjà être tirées.

II existe environ 99,5\% d'identité entre les deux génomes.

En admettant l'hypothèse d'une divergence homme-chimpanzé il y a 6,5 millions d'années, et en utilisant pour faire le calcul de la datation une combinaison de divergence des temps et de vraisemblance, S. Pääbo et al. émettent, prudemment, quelques propositions: Homo sapiens et Homo neanderthalensis auraient divergé il y a environ entre 465000 à 569000 ans, la date la plus probable étant aux alentours de 516000 ans. II apparaîtrait que les Néandertaliens proviendraient d'une très petite population d'environ 3000 individus. Cette estimation correspond à celle de la population ancestrale humaine permettant d'expliquer la quantité de diversité génétique observée dans les populations vivant de nos jours.

- E.M. Rubin et al. n'ont recueilli qu'une faible quantité d'ADN : $65000 \mathrm{pb}$ seulement. Mais ils ont réussi à obtenir 29 des 35 gènes qu'ils escomptaient, ce qui montre l'avantage de la «métagénomique » à recueillir des régions d'intérêt. Leur estimation de la divergence entre les deux espèces est grossiè- 
rement la même : entre 120000 et 670000 ans, le plus probable étant vers 370000 ans.

Ces séquences du passé sont désormais déposées au EBI (European Bioinformatics Institute) et à GenBank.

\section{Des traces de leurs amours?}

Savoir que l'homme de Néandertal n'était pas notre ancêtre fut en son temps une surprise. Puis on s'habitua à faire la différence entre les hommes de Cro-Magnon et ceux de Néandertal, ces imposants chasseurs, gros mangeurs de viande $[14]^{2}$. Sans oublier qu'ils semblaient avoir des rites funéraires (y compris l'incinération) [15], alors qu'on croyait le culte des morts spécifique des sociétés humaines et du sentiment qu'ont les hommes modernes de leur présence au monde.

Mais apprendre que ces deux Homo avaient coexisté pendant 5000 ans (en Europe) à 10000 ans (en Afrique) [16, 17] fut une révélation plutôt dérangeante!

Aussitôt, elle donna lieu à des spéculations. Au cours de cette cohabitation millénaire, ont-ils confronté leurs coutumes et échangé leur savoir-faire ? Ont-ils laissé des traces dans le pool génique de l'homme moderne? En d'autres termes: Did they mate ? [18].

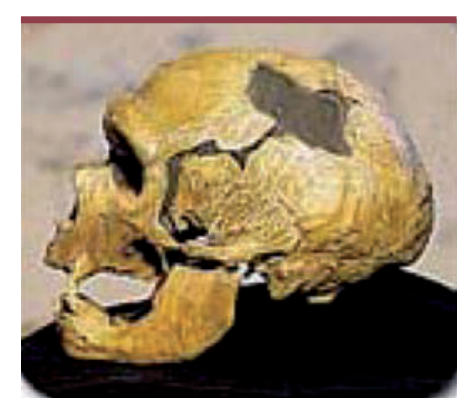

Crâne de l'homme de la Chapelleaux-Saints (๔ Musée de I'Homme de Néandertal).

Si rien ne transparaît dans les résultats de $\varepsilon . M$. Rubin, pour le groupe de Leipzig, la réponse serait positive. En utilisant HapMap et d'autres banques humaines de SNP (single nucleotide polymorphism), S. Pääbo et al. ont constaté que dans $30 \%$ des cas, Néandertal avait l'allèle humain, et non pas l'allèle ancestral (à savoir celui du chimpanzé). Or, l'ancienneté de la séparation des deux taxons est trop importante pour trouver un tel pourcentage. Le fait que le chromosome $X$ de Néandertal ait un degré de divergence plus important que celui des autosomes tendrait à montrer que les croisements auraient eu lieu de façon unilatérale: les humains mâles auraient engendré des enfants avec des femmes néandertaliennes. À l'inverse, dans un récent article [19], B.T. Lahn et al. semblent suggérer le contraire: le gène de la microcéphaline ( $M C P H I)$, supposé

${ }^{2}$ Environ 4500 à 5000 calories/j, soit 2 kg de caribou selon les estimations faites d'après la reconstitution à partir du spécimen bien conservé de La Ferrassie [14]. réguler la taille du cerveau chez l'homme, posséderait de nombreux haplogroupes; parmi ceux-ci, l'haplogroupe $D$, très ancien, aurait été réintroduit récemment (il y a environ 37000 ans) chez les humains modernes par... qui sait?: Néandertal.

\section{Restons prudents}

Ces travaux, au demeurant passionnants, n'en sont encore qu'à leurs balbutiements. La séparation des deux taxons ne vaut que si l'on tient pour acquis la datation de la divergence avec le chimpanzé (6,5 millions d'années). Or celle-ci est controversée. L'ADN de Néandertal a pu subir des dénaturations. Et des contaminations avec l'ADN humain sont toujours à craindre. De plus, les séquences retrouvées ne le sont que par référence aux séquences humaines qui, seules, permettent de les assembler. II y a loin de cet ouvrage de patchwork - qui commence à peine - à la véritable « mise en lettres » d'un génome à partir d'un ADN intact d'un sujet vivant.

Qui plus est, afin de poursuivre le travail, les autres spécimens éventuellement disponibles ne sont pas en aussi bon état de conservation. En effectuant une reconstruction paléoclimatique, des chercheurs britanniques considèrent qu'en Europe, dans 9 sites seulement sur 39 , les fossiles ont pu conserver un ADN analysable, les autres étant inutilisables [20].

Quant à l'héritage que nous aurions laissé à nos cousins avant qu'ils disparaissent corps et biens (ou inversement), il fera les délices d'une certaine presse «à sensation» bien avant que nous en ayons confirmation (ou infirmation). Mais l'impatience est grande de connaître la séquence de certains gènes, comme celle de $M C P H 1$ déjà cité, ou de FOXP2, qui diffère chez I'homme des autres espèces et semblerait impliqué chez lui dans la parole et le langage [21].

Les techniques vont encore progresser. Svante Pääbo - qui a eu le prix Louis-Jeantet de médecine l'an passé - désire poursuivre dans cette voie. Souhaitons-lui bonne chance! $\diamond$ Premisses of the Neanderthal genome

\section{REMERCIEMENTS}

À Bertrand Jordan pour son aide et ses conseils.

\section{RÉFÉRENCES}

1. Beauval C. Difficile cohabitation entre hyènes des cavernes et hommes de Neandertal il y a 40000 ans. Med Sci (Paris) 2005 ; 11: 901-2.

2. Orlando L, Hänni C. Du nouveau pour I'ADN ancien. Med Sci (Paris) 2000 ; $16: \mid-X V I$.

3. Higuchi RC, Wrischnik LA, Oakes $\varepsilon$, et al. Mitochondrial DNA of the extinct quagga: relatedness and extend of post-mortem change. J Mol Evol 1987 ; 25 : 283-7.

4. Rogan P, Salvo JJ. Studies of nucleic acids from ancient remains. Yearbook Phys Anthropol $1990 ; 33: 195-214$.

5. Krings M, Stone A, Schmitz RW, et al. Neanderthal DNA sequence and origin of modern humans. Cell $1997 ; 90: 19-30$.

6. Krings M, Geisert H, Schmitz RW, et al. DNA sequence of the mitochondrial hypervariable region II from the neanderthal type specimen. Proc Natl Acad Sci USA $1999 ; 96: 5581-5$.

7. Orlando $L$, Darlu P, Toussaint $M$, et al. Revisiting Neandertal diversity with a 100,000 year old mtDNA sequence. Curr Biol $2006 ; 16$ : R400-2. 
8. Gilgenkrantz S. Le dernier repas d'Ötzi. Med Sci (Paris) $2003 ; 19: 30$.

9. Green RE, Kranse J, Ptak SE, et al. Analysis of one million base pairs of Neanderthal DNA. Nature 2006 ; $444: 330-6$.

10. Noonan JP, Coop G, Kudaravalli D, et al. Sequencing and analysis of Neanderthal genomic DNA. Science 2006 ; $314: 1113-8$.

11. Marguelies M, Egholm M, Altman WE, et al. Genome sequencing in microfabricated high-density picolitre reactors. Nature $2005 ; 437: 376-80$.

12. Tringe SG, Von Mering C, Kobayashi A, et al. Comparative metagenomics of microbial communities. Science $2005 ; 308: 554-7$.

13. Noonan JP, Hofreiter M, Smith D, et al. Genomic sequencing of Pleistocene cave bears. Science 2005; 309: 597-9.

14. Culotta $\varepsilon$. Calories count reveals Neandertals outate hardiest modern hunters. Science 2005 ; 307: 840-1.

15. Pettitt PB. The Neanderthal dead: exploring mortuary variability in middle palaeolithic Eurasia. Before Farming 2002; 1 : 1-19.

16. Gravina B, Mellars P, Ramsey CB. Radiocarbon dating of interstratified Neanderthal and early modern human occupations at the Chatelperronian typesite. Nature 2005 ; $438: 51-6$.

17. Labie D. Jusqu'à quand l'homme de Neandertal a-t-il vécu sur terre? Med Sci (Paris) 2006 ; $22: 1046$.

18. Pennisi $\varepsilon$. The dawn of stone age genomics. Science $2006 ; 314: 1068-70$.

19. Evans P, Mekel-Bobrov N, Vallender EJ, et al. Evidence that adaptative allele of the brain size gene microcephalin introgressed into Homo sapiens from archaic homo lineage. Proc Natl Acad Sci USA 2006 ; 103: 18178-83.

20. Smith $\mathrm{Cl}$, Chamberlain AT, Riley MS, et al. Not just old but old and cold. Nature 2006 : 410 : 771-2.

21. White SA, Fisher SE, Geschwind DH, et al. Singing mice, songbirds, and more: models for FOXP2 function and dysfunction in human speech and language. J Neurosci 2006 ; 26 : 10376-9.

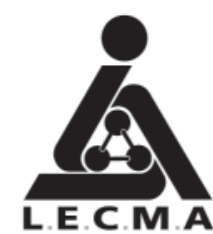

Ligue

Européenne

Contre la

Maladie d'Alzheimer

\section{ApPel aUX bourses POUR La RECHERCHE BiOMÉDICALE ET CLINIQUE FONDAMENTALE SUR LA MALADIE D'ALZHEIMER}

La Ligue Européenne Contre la Maladie d'Alzheimer (LECMA) octroie des bourses pour la recherche scientifique dans le domaine de la maladie d'Alzheimer. Sont acceptées les demandes de scientifiques d'institutions françaises.

Les approches innovantes et révolutionnaires sont encouragées. Les candidats doivent avoir une expérience dans le domaine de la recherche sur la maladie d'Alzheimer. Les jeunes chercheurs doivent être associés à un laboratoire expérimenté en matière de recherche dans le domaine de la maladie d'Alzheimer. Les chercheurs d'un même laboratoire peuvent faire leur demande pour autant que les projets de recherche proposés ne se chevauchent pas.

Les demandes de bourse standard sont acceptées jusqu'à concurrence de $€ \mathbf{8 0 0 0 0}$ pour deux ans avec un maximum de $€ 40000$ pour un an.

Les demandes de bourse pour des projets pilotes par de jeunes chercheurs sont acceptées jusqu'à concurrence de $\boldsymbol{€} \mathbf{4 0 0 0 0}$ pour deux ans avec un maximum de $€ 20000$ pour un an. Seuls les jeunes chercheurs avec une thèse de $\mathrm{PhD}$ et un maximum de 6 ans d'expérience en recherche scientifique sont éligibles.

Les subventions peuvent être utilisées pour couvrir les frais depersonnel, d'équipement et de fournitures. Elles seront mises à disposition en novembre 2007.

Les subventions sont octroyées sur base du mérite scientifique du projet de recherche proposé et de son utilité pour améliorer notre compréhension de la maladie d'Alzheimer. Les demandes seront examinées par le comité consultatif scientifique européen, composés de pairs placés sous la présidence du Dr Frédéric Checler.

Les demandes doivent être rentrées par e-mail (sous forme de fichier Word) à e.wiese@alzheimer-forschung.de et 5 exemplaires par la poste, (un original et quatre copies certifiées conformes), au plus tard le 16 avril 2007, date de la poste faisant foi, à l'adresse suivante:

\section{Ligue Européenne Contre la Maladie d'Alzheimer 21, Place de la RépubliQue, 75003 Paris}

Les formulaires de candidatures et des informations détaillées seront disponibles en janvier 2007 le site Internet: www.MaladieAlzheimer.fr

Pour toute question, veuillez prendre contact avec Dr Ellen Wiese, Research Grant Manager au numéro +49-211-86206621 ou par e-mail: e.wiese@alzheimerforschung.de.

Depuis sa création en 2005, LECMA a soutenu 6 projets de recherche pour un montant de $340000 €$

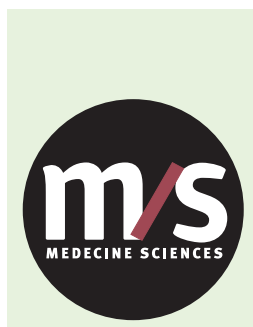
Tarifs d'abonnement M/S - 2007
Abonnez-vous
à Médecine/Sciences

> Depuis 20 ans, grâce à $\mathrm{m} / \mathrm{s}$, vous vivez en direct les progrès des sciences biologiques et médicales

Bulletin d'abonnement page 40 dans ce numéro de $\mathrm{m} / \mathrm{s}$
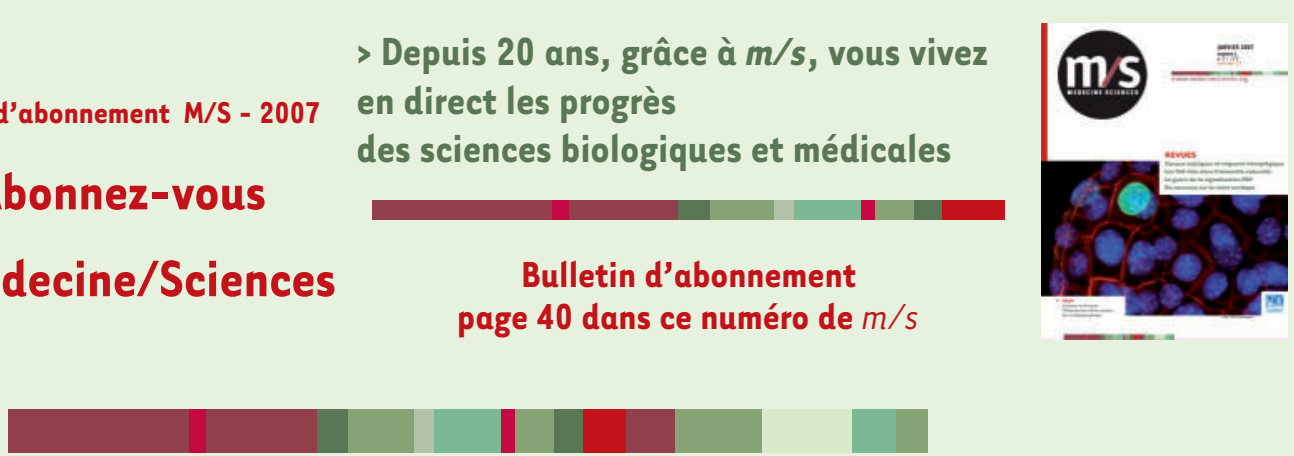